ISSN 0103-7013

Psicol. Argum., Curitiba, v. 29, n. 66, p. 373-381, jul./set. 2011

Licenciado sob uma Licença Creative Commons

\title{
Comunicação médico-cuidador-paciente em onco-hematologia pediátrica: Perspectivas e desafios
}

\author{
Doctor-caregiver-patient communication in pediatric \\ onco-hematology: Perspectives and challenges
}

\section{Marina Kohlsdorf ${ }^{[a]}$, Eliane Maria Fleury Seidl ${ }^{[b]}$}

[a] Doutoranda pela Universidade de Brasília (UnB), psicóloga colaboradora no Hospital Universitário de Brasília (HUB), Brasília, DF - Brasil, e-mail: marinak@unb.br

[b] Doutora pela Universidade de Brasília (UnB), professora adjunta IV da Universidade de Brasília (UnB), Brasília, DF - Brasil, e-mail: seidl@unb.br

\section{Resumo}

Apesar dos recentes avanços técnicos na assistência ao paciente pediátrico, a literatura ainda indica a importância de melhorias na interação comunicativa envolvendo médicos, cuidadores e crianças. Neste estudo, são apresentados aspectos relevantes relativos à comunicação no atendimento pediátrico, em especial as características e os desafios deste processo de interação no contexto onco-hematológico, por se tratar de um tratamento potencialmente estressante e de longa duração. De modo especial, a satisfação com a qualidade da comunicação tem sido apontada como elemento essencial no cuidado pediátrico. Destaca-se ainda a necessidade de maior inclusão da criança na comunicação durante os atendimentos, bem como outros aspectos importantes, referidos pelos cuidadores, que possam proporcionar uma melhor qualidade da interação. Ao fim deste estudo, são apresentadas sugestões para pesquisas e intervenções psicossociais referentes à comunicação entre médico, cuidador e paciente pediátrico, que possam promover a diminuição de custos comportamentais geralmente associados ao tratamento do câncer na infância.

Palavras-chave: Comunicação. Câncer. Psicologia pediátrica. 


\begin{abstract}
Despite the recent technical advances in assisting pediatric patients, the literature on this area still indicates the importance of improving the communicative interaction between physicians, caregivers, and children. In this study, relevant aspects related to communication in the pediatric service are presented, specially characteristics and challenges of the interactive process in an onco-hematological context, given the fact that it is a potentially stressful and long-term treatment. The satisfaction with the quality of communication has been particularly pointed out as an essential element in pediatric care. The need to increase the child's participation in the communication process during pediatric consultations, and also other relevant features referred by caregivers, that may promote a higher interaction quality, are also highlighted here. At the end of this paper, suggestions for research and intervention, related to communication between physicians, caregivers, and patients, are presented, which could promote the decrease in behavioral costs usually associated to pediatric cancer treatment.
\end{abstract}

Keywords: Communication. Cancer. Pediatric psychology.

\section{Introdução}

Estabelecida na década de 1970, a psicologia pediátrica pode ser caracterizada como uma subárea da psicologia da saúde, de intervenção multidisciplinar, que focaliza comportamentos determinantes e condicionantes do processo saúde-doença em uma faixa etária específica, a saber, entre 0 e 18 anos de idade (Viana \& Almeida, 1998). Nesse contexto, torna-se fundamental implementar ações preventivas ao desenvolvimento de patologias e dificuldades psicossociais e compreender como pacientes, familiares e profissionais de saúde lidam com questões relacionadas ao processo saúde-doença.

Uma área de recente interesse para investigação em psicologia pediátrica refere-se aos processos de interação e comunicação estabelecidos entre médicos, familiares e a criança em tratamento (Howells \& Lopez, 2008; Mendonça, 2007). Tates e Meeuwesen (2001) e ainda Sobo (2004) destacam que a comunicação envolve aspectos relativos à explicação sobre diagnóstico, construção de uma interação interpessoal baseada em aspectos afetivos, qualidade da troca de informações entre os participantes e negociação sobre decisões pertinentes ao tratamento de forma colaborativa e compartilhada. Os autores destacam ainda que uma interação comunicativa eficiente em contextos pediátricos deve ser pautada tanto em aspectos técnico-instrumentais quanto afetivo-emocionais.

O investimento na qualidade dos serviços prestados à população pediátrica (com focalização em pacientes e respectivos cuidadores primários ${ }^{1}$ ) pode ser considerado uma ação preventiva em assistência à saúde, possibilitando minimizar ou evitar dificuldades futuras, tanto em caráter biofisiológico quanto psicossocial. Este trabalho apresenta aspectos teóricos sobre o estudo da comunicação em contextos pediátricos, especialmente em relação às interações entre médico, cuidador e criança durante atendimentos e consultas e focalizando a comunicação associada a tratamento onco-hematológico, que representa atualmente um dos maiores desafios para a atuação em psicologia pediátrica.

\section{Concepçóes sociais sobre a infância}

Durante a Idade Média e até o fim do século XVIII, a sociedade percebia as crianças como

1 Termo utilizado para designar o principal indivíduo responsável pelos cuidados básicos ao paciente, acompanhando consultas, exames e internações, definindo decisões sobre o tratamento, fornecendo suporte emocional e administrando práticas educativas. Geralmente, cuidadores pediátricos são os pais da criança, embora familiares e pessoas sem grau de parentesco também possam exercer esse papel (Cunha, 2007). 
adultos em miniatura, desprovidas de subjetividade ou peculiaridades, e somente a partir de estudos sobre a infância em áreas como a sociologia, psicologia, pediatria e educação, essas concepções começaram a ser modificadas (Kennedy, 2000). Mesmo com as mudanças de ordem social, política e ideológica nos últimos séculos, a influência dessas representações ainda é perceptível nos tempos atuais, determinando o papel da infância na sociedade e relegando a crianças uma atuação meramente passiva e receptiva.

Kennedy (2000) destaca que as crianças interpretam os fatos de maneira diferente daquela exercida pelos adultos, formulando vivências, compreensões e experiências bastante peculiares e que não refletem uma versão meramente rudimentar da concepção dos adultos. Torna-se necessária uma mudança paradigmática nas concepções sobre a infância, pois mesmo diante dos progressos nos estudos sobre o desenvolvimento infantil, em inúmeras áreas de conhecimento ainda é perceptível a visão de mundo centralizada no adulto e a interpretação das vivências infantis segundo o ponto de vista adultocêntrico, que não reflete de forma fiel as experiências vivenciadas pela criança nem sua percepção sobre o mundo. De modo especial, investigar e compreender como a população pediátrica vivencia processos de saúde-doença pode promover intervenções mais eficientes e melhorias significativas na qualidade de vida dessa população e de familiares envolvidos.

\section{A concepçáo da infância no contexto de atendimento pediátrico}

Segundo Patenaude e Last (2001), o aumento da atenção à saúde da criança e do adolescente tem determinado consequências importantes para a sobrevivência e qualidade de vida dessa população, a partir do aumento nos índices de sobrevida, diminuição da mortalidade infantil e redução da incidência de patologias antes preocupantes, tais como doenças infectocontagiosas. O progresso na atenção à população pediátrica pode ser atribuído a melhores técnicas para diagnóstico e tratamento de enfermidades, investimento em programas preventivos, ampliação do acompanhamento pré, peri e pós-natal e aumento de ações em políticas públicas para controle da morbimortalidade infanto-juvenil. O investimento na atenção básica à criança contribui para modificar o perfil de adoecimento e mortalidade dessa população e destaca a importância de compreender como crianças e adolescentes vivenciam o diagnóstico e tratamento de patologias agudas e crônicas, para implementar intervenções psicossociais que promovam melhor qualidade de vida durante processos de diagnóstico e tratamento de doenças pediátricas.

No contexto de atenção à saúde infantil, a literatura indica que as ações assistenciais ainda refletem as ideias historicamente associadas à concepção da infância, relegando ao paciente um papel restrito e passivo. Outsubo e Becker (2005) destacam que o atendimento na faixa etária pediátrica não oferece condições específicas de assistência e que a atenção à população infantil deveria ser pautada em especificidades, na medida em que não se trata de um atendimento a adultos em miniatura, mas a indivíduos em pleno processo de desenvolvimento, que possuem subjetividade, particularidades e singularidades. Os autores destacam ainda que o estudo das interações comunicativas em contexto pediátrico evidencia a exclusão do paciente de seu próprio processo de diagnóstico e tratamento, contribuindo para a centralização do atendimento no profissional de saúde e restringindo a participação da criança na interação durante consultas e atendimentos.

\section{Comunicação em contextos pediátricos: papéis do cuidador e da criança}

Em contextos de atenção à saúde pediátrica, o atendimento é necessariamente caracterizado pela presença, no mínimo, de um profissional de saúde, do paciente e de um cuidador, na maioria das vezes a mãe da criança, que geralmente é designada como a principal responsável pelos cuidados em saúde e práticas educativas parentais (Sobo, 2004; Tates \& Meeuwesen, 2001). Portanto, o contexto de atendimento pediátrico envolve a interação mínima em tríades (médico-acompanhante-paciente), situação bastante diferente das consultas a pacientes adultos em que apenas uma díade, em geral, participa dos processos de comunicação. A dinâmica interativa de uma tríade é fundamentalmente diferente daquela estabelecida entre dois indivíduos, para a qual os profissionais de saúde são preparados durante sua formação; como consequência, especialmente em contexto pediátrico, a interação tende a se estabelecer 
de modo diádico (médico-acompanhante; médico-paciente ou acompanhante-criança), excluindo um participante da comunicação (Howells \& Lopez, 2008; Lienard et al., 2008; Nobile \& Drotar, 2003; Nova, Vegni \& Moja, 2005; Tates \& Meeuwesen, 2001; Tates, Elbers, Meeuwesen \& Bensing, 2002).

A literatura sobre aspectos psicossociais no atendimento indica em consenso que as consultas são centradas no médico, que administra o atendimento, orienta perguntas ao cuidador, estabelece os assuntos abordados e determina, portanto, as condições para a comunicação (Dowset et al., 2000; Hayutin, Reed-Knight, Blount, Lewis \& McCormick, 2009). Fatores como a idade da criança, tempo de experiência do médico, motivo da consulta e características sociodemográficas do cuidador e do paciente influenciam a qualidade da interação em consultas, mas a comunicação ocorre quase exclusivamente entre médico e cuidador: o paciente pediátrico é excluído do processo de interação comunicativa (Dulmen, 2004; Hayutin et al., 2009; Nobile \& Drotar, 2003; Nova et al., 2005).

Um foco de investigação importante relativo à comunicação em contextos pediátricos refere-se à satisfação dos cuidadores com a qualidade da interação. De acordo com os trabalhos de Dowset et al. (2000) e ainda Howells e Lopez (2008), uma comunicação satisfatória envolve centralização da consulta no paciente e no cuidador, menor dominância do médico na condução do atendimento e promoção de acolhimento a dúvidas e preocupações dos cuidadores. Além disso, também foi destacada a inclusão de perguntas, por parte do médico, sobre aspectos psicossociais relativos à vivência do tratamento, promoção de um ambiente amigável e acolhedor, demonstração de atenção em relação às preocupações dos pais e qualidade das informações recebidas.

A qualidade da interação comunicativa entre médicos e cuidadores tem consequências diretas para o sucesso do tratamento, pois proporciona maior satisfação dos cuidadores com o serviço e promove melhores cuidados ao paciente, além de maior retenção de informações, maior envolvimento e colaboração com o tratamento, melhor adesão a recomendações médicas, maior controle de cuidados, menos retornos ambulatoriais, diminuição na frequência de internações e maior discussão de aspectos psicossociais que influenciam o tratamento (Hart, Crotar, Gori \& Lewin, 2006; Hart, Drotar \&
Scholle, 2007; Howells \& Lopez, 2008; Mendonça, 2007; Nobile \& Drotar, 2003).

\section{A participaçáo da criança durante o atendimento pediátrico}

A comunicação em contextos pediátricos é fundamentada na díade médico-cuidador: mesmo quando o profissional se dirige à criança, geralmente o conteúdo da comunicação reflete aspectos lúdicos e as perguntas direcionadas à criança são comumente mediadas pelo cuidador (Jongudomkam, Aungsupakom \& Camfield, 2006; Nova et al., 2005; Tates et al., 2002). Os adultos têm papel principal na interação do atendimento pediátrico e a estrutura das consultas não permite a real inserção do paciente pediátrico. Consequentemente, as oportunidades de participação infantil são limitadas pelos médicos e pais, dificultando assim a atuação da criança em negociações, explicações e decisões sobre seu próprio tratamento (Nova et al., 2005; Tates \& Meeuwesen, 2001; Tates et al., 2002). Cabe ainda destacar que a escassez de incentivo e suporte dos cuidadores e médicos à participação efetiva da criança em consultas reflete uma coconstrução social e institucional, refletindo a assimetria cultural historicamente determinada na relação entre adultos e crianças (Nova et al., 2005; Outsubo \& Becker, 2005; Tates \& Meeuwesen, 2000; Tates \& Meeuwesen, 2001; Tates et al., 2002).

Estudos na literatura sobre atendimento pediátrico indicam que pacientes a partir de quatro anos de idade são capazes de perceber e nomear sintomas, apontar órgãos atingidos, procurar causas para a enfermidade (muitas vezes atribuindo culpabilização a si mesmos pelo adoecimento), perceber alterações no cotidiano em decorrência do tratamento (como mudanças na rotina escolar e familiar) e relacionar prejuízos funcionais advindos da patologia, além de lidar de forma atuante e crucial diante dos desafios e dificuldades intrínsecos à sintomatologia e tratamento (Howells \& Lopez, 2008; Jongudomkam et al., 2006; Nova et al., 2005; Outsubo \& Becker, 2005; Tates \& Meeuwesen, 2001). O trabalho de Nova et al. (2005) defende que crianças de dois anos de idade já poderiam ter maior inclusão e participação em consultas médicas, para incentivar habilidades de compreensão sobre a patologia, eliminar crenças disfuncionais e fundamentar habilidades futuras de responsabilidade no tratamento, contribuindo também para alterações 
comportamentais de médicos e cuidadores na forma de conduzir o atendimento.

Destaca-se, portanto, a necessidade de modificar as práticas comunicativas em atendimento pediátrico, pois a criança deve ter participação efetiva no processo terapêutico e em negociações sobre o tratamento. As práticas tradicionalmente estruturadas ainda impõem a pacientes pediátricos o papel de meros espectadores, para assimilação passiva de informações e planejamentos. Cabe ao médico e aos pais, como participantes dominantes da interação comunicativa, possibilitar um ambiente promotor do desenvolvimento da criança, que certamente proporcionará menores custos comportamentais associados ao tratamento, tanto para profissionais de saúde quanto para cuidadores e pacientes. Os estudos de Howells e Lopez (2008), Nobile e Drotar (2003), Nova et al. (2005) e ainda Tates e Meeuwesen (2000) indicam que a maior inclusão do paciente pediátrico na interação comunicativa certamente ocasiona consequências benéficas para o atendimento, tais como: maior satisfação da criança com o serviço de saúde, maior colaboração com o tratamento, ajuste do atendimento a necessidades e características do paciente, melhor qualidade do autocuidado, melhor adesão ao tratamento, maior retenção de informações por parte da criança, menores retornos do paciente ao serviço e menos internações.

\section{$O$ contexto de tratamento em onco-hematologia pediátrica}

O câncer na infância corresponde a um grupo de mais de duzentas doenças, de etiologia multifatorial, cuja particularidade está na multiplicação desordenada de células disfuncionais (McGrath, Paton \& Huff, 2005). O tratamento antioneoplásico representa diversos desafios para pacientes e familiares - modificações na rotina, ansiedade pela resposta clínica ao tratamento, crenças disfuncionais sobre a patologia, mudanças e desorganizações da dinâmica familiar são comumente vivenciadas. A criança que vivencia um episódio de câncer precisa lidar com repetidos procedimentos médicos invasivos, efeitos colaterais da medicação, interrupção da rotina escolar, suspensão de algumas atividades de lazer, alteração da dieta, mudanças na autoimagem e autoconceito, incerteza em relação à evolução do tratamento, dor e perdas que se prolongam por um extenso período e podem prejudicar a manutenção de relacionamentos pessoais e sociais (McGrath et al., 2005; Patenaude \& Last, 2001; Shiota, Santos \& Miyazaki, 2004).

Quando comparadas a outras doenças prevalentes na população infantil, as patologias onco-hematológicas apresentam custos comportamentais bastante intensos, em razão do tratamento agressivo e extenso (em geral, entre 6 e 24 meses, a depender da patologia), dos efeitos colaterais da medicação quimioterápica, da repetição de procedimentos invasivos, das alterações na rotina e dinâmica familiar e das incertezas em relação ao sucesso do tratamento. Uma dificuldade adicional nesse contexto de tratamento inclui a etiologia multifatorial e relativamente desconhecida do câncer pediátrico, a complexidade dos protocolos terapêuticos e as representações socialmente associadas às neoplasias, que incluem vivências de perdas, sofrimento e morte. Esses aspectos podem contribuir para aumentar a aversividade associada ao tratamento antineoplásico e dificultar a comunicação entre médico, cuidador e paciente no contexto de atendimento.

Patenaude e Last (2001) indicam ainda que, desde 1970, a combinação de técnicas quimioterápicas e cirúrgicas, o investimento em ações preventivas, o incentivo ao acompanhamento precoce e surgimento de novas técnicas diagnósticas possibilitaram um aumento importante da sobrevivência de crianças ao câncer, indicando a necessidade de mais intervenções e pesquisas nessa área, especialmente relacionadas à vivência de cuidadores e pacientes. Além disso, tornam-se essenciais intervenções sistemáticas que minimizem o impacto da terapêutica antineoplásica, além da investigação de quais fatores estão associados à diminuição de custos comportamentais associados ao tratamento. Nesse contexto, a qualidade da interação comunicativa pode influenciar de forma significativa o impacto do diagnóstico e tratamento do câncer na infância, contribuindo para minimizar as dificuldades inerentes ao processo de adoecimento e facilitar a adaptação mais rápida de pacientes e cuidadores aos desafios impostos pelo tratamento.

\section{A comunicaçáo em contexto onco-hematológico pediátrico}

Segundo Fisher (2005) e Sobo (2004), no contexto de atendimento onco-hematológico, a 
consulta médica segue o mesmo padrão de outras especialidades pediátricas, com a focalização da interação médico-cuidador e a exclusão do paciente na interação comunicativa. Os autores indicam ainda que as informações sobre o tema são, em geral, muito complexas e permeadas pela carga emocional e incerteza que caracterizam o diagnóstico e tratamento antineoplásico, propiciando condições para uma comunicação ineficiente e para consequências prejudiciais à vivência do tratamento (manifestações de ansiedade, adesão insatisfatória às recomendações terapêuticas, sobrecarga de cuidados e baixa retenção de informações).

$\mathrm{Na}$ literatura, são referidos poucos trabalhos que abordaram o contexto de comunicação em onco-hematologia pediátrica. Em estudo exploratório, Miller, Drotar, Burant e Kodish (2005) indicaram que uma comunicação eficiente entre médicos e cuidadores promove uma atuação mais ativa destes últimos no tratamento, além de maior confiança na equipe de saúde, melhor retenção de informações sobre diagnóstico e planejamento terapêutico, diminuição de relatos de ansiedade e aumento da percepção de controle sobre a situação. Os autores destacam ainda que algumas características sociodemográficas dos cuidadores (raça e condição socioeconômica) foram importantes preditores da qualidade da comunicação entre cuidadores e médicos: pais com piores condições econômicas receberam menor quantidade de informações.

O estudo de Mendonça (2007) sobre a comunicação de diagnóstico em onco-hematologia pediátrica indicou resultados igualmente relevantes. A autora destaca que, nesse trabalho, os médicos também exerceram relação de dominância ao dirigir a consulta pediátrica, estabelecendo quais assuntos foram focalizados e limitando a abordagem de aspectos afetivos e psicossociais importantes, sendo que a participação dos cuidadores, de forma geral, ficou restrita a responder os questionamentos dos médicos. O repasse de informações por parte dos médicos foi caracterizado pela adaptação da linguagem técnica para o contexto do paciente e cuidador, priorização da atenção ao cuidador, inadequação de perguntas para verificar a compreensão de informações (uso de perguntas fechadas, com possibilidades de resposta apenas "sim" ou "não") e incapacidade para lidar de forma satisfatória com respostas emocionais do cuidador e do paciente. Além disso, os cuidadores referiram que o momento da consulta é insuficiente para compreender aspectos relevantes da doença, pois o impacto emocional do diagnóstico e a incerteza em relação ao sucesso do tratamento prejudicam a retenção de informações repassadas durante o atendimento.

Clarke e Fletcher (2003) investigaram a satisfação de cuidadores com a comunicação estabelecida em contexto onco-hematológico pediátrico, bem como sua relação com custos comportamentais e vivências do tratamento. Os autores indicaram relatos por parte dos pais caracterizados por desconfiança, em alguns momentos, em relação à equipe médica e omissão de informações importantes, além de queixas sobre pouco suporte social e reduzida habilidade interativa dos médicos diante de respostas emocionais e preocupações dos cuidadores. Os pais destacaram ainda o recebimento de informações contraditórias em relação a recomendações de cuidados, falta de oportunidades para referir dúvidas, incertezas, preocupações e receios, respostas emocionais de raiva e frustração quando as preocupações e questionamentos eram ignorados e ainda um desequilíbrio na quantidade de informações recebidas (algumas vezes, a informação era repassada em demasia, outras vezes faltavam elementos importantes para a compreensão da informação). Uma comunicação eficiente foi caracterizada pelo fornecimento adequado de suporte social por parte dos médicos, clareza nas informações e acolhimento durante as consultas. O estudo indicou também que, quando os pais encontram um ambiente acolhedor a suas preocupações, conseguem desenvolver e manter bons relacionamentos com a equipe de saúde, promovendo assim melhores condições para lidar com as adversidades do tratamento, indicando maior percepção de suporte social, maior satisfação com o serviço e diminuição de sobrecarga e de custos comportamentais associados ao tratamento.

\section{Consideraçóes finais}

$\mathrm{O}$ atendimento em pediatria ainda prioriza a interação entre médico e cuidadores e reduz a participação do paciente a níveis mínimos, mesmo diante de mudanças sociopolíticas sobre concepções relativas à infância e estudos advindos de campos como a sociologia, psicologia e educação. Em contexto onco-hematológico pediátrico, caracterizado pelos elevados custos comportamentais associados 
ao diagnóstico e tratamento, as pesquisas constatam o aspecto dualista da interação entre médico e cuidador, destacando a satisfação dos pais com a qualidade da interação, mas sem referências sobre intervenções sistematizadas que promovam uma comunicação mais eficiente e inclusiva da criança nesse contexto. $\mathrm{O}$ investimento na melhoria da interação comunicativa em onco-hematologia pediátrica torna-se, portanto, elemento fundamental na assistência em saúde, a partir de ações direcionadas tanto à interação com os cuidadores quanto à participação do paciente pediátrico em seu próprio tratamento. Cabe destacar que a qualidade de comunicação no tratamento do câncer na infância é um preditor importante sobre o sucesso terapêutico, influenciando diretamente a maneira como cuidadores e pacientes lidam com as dificuldades de diagnóstico e tratamento (Mendonça, 2007; Patenaude \& Last, 2001).

São necessárias mais pesquisas e intervenções psicossociais que priorizem o processo de comunicação médico-cuidador-paciente nos atendimentos e facilitem a inclusão do paciente na interação triádica, considerando que a criança em protocolo antineoplásico é um participante ativo em seu tratamento, ao lidar com alterações escolares, cotidianas e em relacionamentos sociofamiliares decorrentes do tratamento, e também ao enfrentar diretamente os procedimentos aversivos e dolorosos (Howells \& Lopez, 2008; Jongudomkam et al., 2006; Nova et al., 2005; Outsubo \& Becker, 2005).

Cabe destacar que promover a inclusão da criança na tríade pediátrica é uma ação preventiva em nível primário e secundário na atenção à saúde pediátrica, aumentando a eficiência do tratamento. É responsabilidade de médicos e cuidadores promover a inclusão da criança na comunicação, criando um ambiente propício ao desenvolvimento infantil e favorecendo a aquisição gradual de senso de responsabilidade sobre sua própria saúde (Nova et al., 2005; Tates \& Meeuween, 2000; Tates et al., 2002). A maior autonomia e participação infantil no processo de decisão sobre seu tratamento representam benefícios importantes: melhor relacionamento com a equipe, ganho de confiança e consequente colaboração com o tratamento, negociação sobre cuidados terapêuticos, melhor adesão ao tratamento, destituição de crenças disfuncionais (como, por exemplo, culpabilização pela patologia), melhor retenção de informações e melhor adaptação a contextos aversivos (Dowset et al., 2000; Howells
\& Lopez, 2008; Mendonça, 2007; Nobile \& Drotar, 2003; Nova et al., 2005; Outsubo \& Becker, 2005; Sobo, 2004).

Autores como Outsubo e Becker (2005) destacam que muitas vezes a compreensão da criança sobre a doença depende mais de informações fornecidas, superando os limites impostos por fases de desenvolvimento. Como consequência, a maior interação da criança na comunicação promove maior autonomia e proporciona um contexto propício para a melhor adaptação do paciente a elementos aversivos do tratamento onco-hematológico, auxiliando também cuidadores e profissionais de saúde a lidar melhor com os desafios impostos pelo tratamento (Howells \& Lopez, 2008; Nobile \& Drotar, 2003).

Além disso, de forma geral, a formação dos profissionais em saúde não inclui a aquisição de habilidades comunicativas para tríades em contexto onco-hematológico pediátrico (Howells \& Lopez, 2008). Os estudos destacaram que as habilidades de interação comunicativa em atendimento devem necessariamente incluir o amparo adequado a respostas emocionais tanto do cuidador quanto do paciente (medo, ansiedade, raiva, culpabilização e incertezas, por exemplo), além da abordagem a aspectos psicossociais importantes e promoção de um contexto acolhedor e propício ao fornecimento de suporte social.

A literatura indica intervenções psicossociais para melhorar habilidades comunicativas no atendimento em saúde, que podem ser adaptadas ao contexto onco-hematológico pediátrico: treinamentos em vídeo, aulas temáticas, oficinas de role play com profissionais especializados, alterações nos currículos de graduação e inclusão de disciplinas específicas, investimento na compreensão da vivência do cuidador, familiares e pacientes, preparação adequada do ambiente terapêutico, treinamento para perceber dicas não verbais durante a consulta, mudanças na abordagem a dúvidas e preocupações de cuidadores e pacientes, incorporação de analogias à linguagem técnica, uso de dicas visuais para explicar o diagnóstico e tratamento, uso de técnicas alternativas para deixar a criança à vontade durante consultas e promoção de possibilidades para negociar prescrições, horários, exames e consultas (BonnaudAntignac, Campion, Pottier \& Supiot, 2009; Bylund et al., 2009; Dulmen et al., 2004; Hart et al., 2006; Hart et al., 2007; Hayutin et al., 2009; Howells \& 
Lopez, 2008; Lienard et al., 2008; Nobile \& Drotar, 2003; Nova et al., 2005).

\section{Sugestóes para futuras pesquisas}

Destaca-se a necessidade de mais pesquisas em contexto onco-hematológico pediátrico que investiguem a interação comunicativa como uma proposição em tríade, além de compreender como elementos na estrutura de atendimento podem prejudicar a comunicação (como a disponibilidade reduzida de tempo para cada consulta, rotatividade do médico atendente, espaço físico inadequado e interrupção da consulta por outros profissionais), conforme indicado por Mendonça (2007). Além disso, variáveis potencialmente mediadoras da comunicação (como estratégias de enfrentamento do cuidador e preferências individuais relativas à quantidade de informação) também devem ser averiguadas, bem como diferenças culturais e variáveis sociodemográficas associadas ao processo de comunicação (Clarke \& Fletcher, 2003; Hayutin et al., 2009; Miller et al., 2005; Nobile \& Drotar, 2003; Sobo, 2004; Tates \& Meeuwesen, 2001).

Outros aspectos que também merecem investigação no processo de comunicação em onco-hematologia pediátrica incluem diferenças em padrões de interação em função do tipo de patologia e tratamento, processo de comunicação em contexto de cuidados paliativos, interação comunicativa com outros profissionais de saúde e investigação longitudinal sobre mudanças na comunicação ao longo do tratamento, a partir de abordagens multimetodológicas e idiográficas. $\mathrm{O}$ processo de comunicação em onco-hematologia pediátrica tem sido apontado como um dos principais preditores de sucesso no tratamento, sendo, portanto, fundamental para garantir a sua qualidade e diminuir os custos comportamentais vivenciados pela criança, seus cuidadores, familiares e profissionais de saúde envolvidos nesse processo (Miller et al., 2005; Sobo, 2004).

\section{Referências}

Bonnaud-Antignac, A., Campion, L., Pottier, P., \& Supiot, S. (2010). Videotaped simulated interviews to improve medical students skills in disclosing a diagnosis of cancer. Psychooncology, 19(9):975-981.
Bylund, C. L., Brown, R., Gueguen, J. A., Diamond, C., Bianculli, J., \& Kissane, D. W. (2010). The implementation and assessment of a comprehensive communication skills training curriculum for oncologists. Psychooncology, 19(6), 583-593.

Clarke, J. N., \& Fletcher, P. (2003). Communication issues faced by parents who have a child diagnosed with cancer. Journal of Pediatric Oncology Nursing, 20(4), 175-191.

Cunha, C. M. (2007). Avaliação transversal da qualidade de vida de cuidadores de crianças e adolescentes com câncer por meio de um instrumento genérico "36 Item Short Form Health Survey Questionnaire” (SF - 36). Dissertação de Mestrado, Universidade Federal de Uberlândia, Uberlândia.

Dowset, S. M., Saul, J. L., Butow, P. N., Dunn, S. M., Biyer, M.J., Findlow, R., et al. (2000). Communication styles in the cancer consultation: Preferences for a patient-centered approach. Psychooncology, 9(2), 147-156.

Dulmen, S. (2004). Pediatrician-parent-child communication: Problem-related ornot?. Patient Education and Counseling, 52(1), 61-68.

Fisher, C. B. (2005). Commentary: SES, Ethnicity and goodness-of-fit in clinician-parent communication during pediatric cancer trials. Journal of Pediatric Psychology, 30(3), 231-234.

Hart, C. N., Drotar, D., Gori, A., \& Lewin, L. (2006). Enhancing parent-provider communication in ambulatory pediatric practice. Patient Education and Counseling, 63(1/2), 38-46.

Hart, C. N., Kelly, K. J., Drotar, D., \& Scholle, S. H. (2007). Parent-provider communication and parental satisfaction with care of children with psychosocial problems. Patient Education and Counseling, 68(2), 179-185.

Hayutin, L. G., Reed-Knight, B., Blount, R. L., Lewis, J., \& McCormick, M. L. (2009). Increasing parentpediatrician communication about children's psychosocial problems. Journal of Pediatric Psychology, 34(10), 1155-1164.

Howells, R., \& Lopez, T. (2008). Better communication with children and parents. Paediatrics and Child Health, 18(8), 381-385. 
Jongudomkam, D., Aungsupakom, N., \& Camfield, L. (2006). The meanings of pain: A qualitative study of the perspectives of children living with pain in northeastern Thailand. Nursing and Health Sciences, 8(3), 156-163.

Kennedy, D. (2000). As raízes do estudo da infância: História social, arte e religião. In W. O. Kohan \& D. Kennedy (Org.). Filosofia e infância: Possibilidades de um encontro (pp. 129-159). Petrópolis: Vozes.

Lienard, A., Merckaert, I., Libert, Y., Delvaux, N., Marchal, S., Boniver, J., et al. (2008). Factors that influence cancer patients' and relatives' anxiety following a three-person medical consultation: Impact of a communication skills training program for physicians. Psychooncology, 17(5), 488-496.

McGrath, P., Paton, M. A., \& Huff, N. (2005). Beginning treatment for pediatric acute myeloid leukemia: The family connection. Issues in Comprehensive Pediatric Nursing, 28(2), 97-114.

Mendonça, M. B. (2007). Análise do processo de comunicação entre médico, acompanhante e paciente em onco-hematologia pediátrica. Dissertação de Mestrado, Universidade de Brasília, Brasília.

Miller, V., Drotar, D., Burant, C., \& Kodish, E. (2005). Clinician-parent communication during informed consent for pediatric leukemia trials. Journal of Pediatric Psychology, 30(3), 219-229.

Nobile, C., \& Drotar, D. (2003). Research on the quality of parent-provider communication in pediatric care: Implications and recommendations. Developmental and Behavioral Pediatrics, 24(4), 279-290.

Nova, C., Vegni, E., \& Moja, E.A. (2005). The physicianpatient-parent communication: A qualitative perspective on the child's contribution. Patient Education and Counseling, 58(3), 327-333.

Outsubo, A. P. N., \& Becker, E. (2005). Crianças com doenças crônicas falam sobre doença: uma pesquisa exploratória. Cadernos de Pós-Graduação em Distúrbios do Desenvolvimento, 5(1), 39-46.

Patenaude, A. F., \& Last, B. (2001). Cancer and children: Where are we coming from? Where are we going? Psychooncology, 10(28), 281-283.
Shiota, C. M., Santos, A. R. R., \& Miyazaki, M. C. O. S. (2004). Problemas de comportamento em crianças com câncer: O papel dos pais. In M.Z. S. Brandão, F. C. S. Conte, F. S. Brandão, Y. K. Ingberman, C. B. Moura, V. M. Silva, et al. (Org.). Sobre comportamento e cognição, 14 (pp. 261-266). São Paulo: ESETEC.

Sobo, E. J. (2004). Good communication in pediatric cancer care: A culturally-informed research agenda. J Pediatr Oncol Nurs, 21(3), 150-154.

Tates, K., \& Meeuwesen, L. (2000). 'Let Mum have her say': Turntaking in doctor-parent-child communication. Patient Education and Counseling, 40(2), 151-162.

Tates, K., \& Meeuwesen, L. (2001). Doctor-parent-child communication: A (re)view of the literature. Social Science and Medicine, 52(6), 839-851.

Tates, K., Elbers, E., Meeuwesen, L., \& Bensing, J. (2002). Doctor-parent-child relationships: A 'pas de trois'. Patient Education and Counseling, 48(1), 5-14.

Viana, V., \& Almeida, J. P. (1998). Psicologia pediátrica: Do comportamento à saúde infantil. Análise Psicológica, 1(16), 29-40.

Recebido: 12/07/2010

Received: 07/12/2010

Aprovado: 21/09/2010 Approved: 09/21/2010 